\title{
Männer auf dem Weg aus der Sozialen Arbeit
}

\author{
Uta Klein \\ Jan Wulf-Schnabel
}

\begin{abstract}
Männer sind in Sozial-, Erziehungs- und Pflegeberufen bekanntlich in der Minderheit. Im Hinblick auf eine geschlechtergerechte Gesellschaft wäre eine größere männliche Präsenz und Erwerbsbeteiligung im weiblich konnotierten Care-Bereich jedoch wichtig. Im Folgenden geht es um den Status quo und die zukünftige Entwicklung der Geschlechterverteilung im Feld der Sozialen Arbeit. Dabei zeigt sich, dass bei wachsender Bedeutung der Sozialen Arbeit Männer in dieser sich entwickelnden Profession immer weniger vertreten sind.
\end{abstract}

\section{Männer in der Minderheit und fern der Basis}

Von der Kinderbetreuung über Erziehung und Bildung, Hilfe und Beratung bis hin zur Pflege richtet sich Soziale Arbeit an beide Geschlechter. Folglich werden sowohl Frauen als auch Männer in der Sozialen Arbeit benötigt. Dieser Sachverhalt steht in einem auffälligen Kontrast zu den Anteilen von Frauen und Männern unter den Professionellen. In den Sozialberufen liegt der Frauenanteil bei rund $77 \%$ (IAB 2006), eine Zahl, die bei Berücksichtigung NichtQualifizierter in der beruflichen Praxis noch höher ausfallen könnte (Cloos/ Züchner 2005, S. 714, 725). Besonders auffällig ist, dass die Beschäftigtenzahl von Frauen zwischen 1999 und 2004 in den Sozial- und Erziehungsberufen insgesamt um 14,8 \% zugenommen hat, bei Männern jedoch nur um 1,6 \% (BMfSFJ 2005, S. 687). Berücksichtigt man nicht die Stundenzahlen, haben vor allem Frauen von den Steigerungen der Beschäftigtenzahlen in den Gesundheits-, Sozial- und Erziehungsberufen profitiert.

In das Zentrum der Aufmerksamkeit ist in den letzten Jahren der Elementarbereich, also die Phase der vorschulischen Erziehung, gerückt. Seit 15 Jahren liegt hier der Anteil der männlichen Beschäftigten bei rund $4 \%$. Kasiske et al. (2006) haben gezeigt, dass dieserAnteil unverändert ist, obwohl sich die Zahl der Beschäftigten zwischen 1990/91 und 2002 in den westdeutschen Einrichtungen um 55 \% erhöht, in den ostdeutschen Bundesländern jedoch nahezu halbiert hat. Ein leichter Rückgang aller in Kindertagesstätten beschäftigten Männer kommt durch die Verringerung des Männeranteils in Ostdeutschland zustande (von 4,1 \% auf 3,7\%). Die Autoren machen auch deutlich, dass der Wert von $4 \%$ - diese Zahl prägt die öffentliche Diskussion - noch nicht einmal das wahre Ausmaß der höchst beunruhigenden männlichen Ferne zum Kind widerspiegelt. Berücksichtigt man nämlich, dass hier neben Erziehungsberufen auch Zivildienstleistende und Hausmeister mitgezählt werden, sinkt der Männeranteil in den „Tätigkeitsarten" Erziehung und frühkindliche Erziehung auf deutlich unter $3 \%$ (Kasiske et al. 2006, S. 20; Rohrmann 2006). Grundlegende Veränderungen gibt es hier nicht, denn die Männeranteile der letzten Jahre weisen nur unregelmäßige, leichte Schwankungen auf (IAB 2006). Gerade vor dem Hintergrund, dass häufig bemängelt wird, im Elementarbereich fehle es an männlichen Identitätspersonen, ist auffällig, dass Kindergärtner, Kinderpfleger und Erzieher seit Jahren signifikant stärker von Arbeitslosigkeit betroffen sind als Frauen in denselben Berufen. Gegenüber den Frauen (9,5\%) lag 2005 die Arbeitslosenquote der Männer mit $18,0 \%$ fast doppelt so hoch. Dieses Phänomen zeigt sich auch in den vorangegangenen Jahren (IAB 2006).

Für die Entwicklung der Geschlechterverhältnisse ist diese Bestandsaufnahme insgesamt verheerend. Denn in der heutigen Gesellschaft wird von Jungen und Männern erwartet, Fürsorge und Bindung in ihre Männlichkeitsentwürfe zu integrieren. Sie können jedoch nicht auf Fürsorgeerfahrungen durch Männer zurückgreifen und es fehlen ihnen ausreichende Vorbilder dafür (King 2000, S. 103).

\section{Akademische Abschlüsse und Tätigkeitsfelder}

Auch im Teilarbeitsmarkt „Sozialarbeit/Sozialpädagogik“ (IAB 2006, Berufsordnung
861 und 862) sind Männer unterrepräsentiert. Differenziert nach Hochschulabschluss ist der Männeranteil unter den erwerbstätigen SozialarbeiterInnen und SozialpädagogInnen mit Fachhochschulabschluss (FH) - nicht überraschend geringer als mit Universitätsabschluss: $33 \%$ gegenüber $40 \%$ (im Jahr 2002). Und er sinkt: 1993 waren noch $38 \%$ der erwerbstätigen SozialarbeiterInnen mit FHAbschluss männlich (Pich 2004). Auch wenn die Datenlage schwer überschaubar ist, weil das Statistische Bundesamt, die Jugendhilfestatistik und der Mikrozensus als Datenquellen unterschiedliche Eingruppierungen vornehmen und zudem die Tätigkeitsbereiche außerordentlich breit sind, so lässt sich doch eine eindeutige Geschlechtersegregation im Berufsfeld erkennen. Die Spaltung verläuft sowohl horizontal (nach Art der ausgeübten Tätigkeit und Einsatzbereichen) als auch vertikal (nach Hierarchie und Eingruppierung).

Pich (2004) unterscheidet unter anderem zwei Tätigkeitstypen. Der Typus der „unmittelbar oder mittelbar personenorientierten Kerntätigkeiten " umfasst: Gesetze anwenden, auslegen, beurkunden/er-

Uta Klein, Prof. Dr., Soziologin, Professorin
an der Fachhochschule Kiel und Privat-
dozentin an der Universität Münster.
Arbeitsschwerpunkte u.a.: Geschlecht und
soziale Ungleichheit, Gleichstellungspolitik
in der EU.
e-mail: Uta.Klein@FH-Kiel.de
Jan Wulf-Schnabel, M.A., Diplom-Sozialwirt,
Lehrbeauftragter an der Fachhochschule Kiel,
Stipendiat der Hans-Böckler-Stiftung im
Masterstudiengang Gender und Arbeit
an der Universität Hamburg. Forschungs-
schwerpunkte: Reorganisation der Freien
Wohlfahrt, Care-Work und Männlichkeit.
e-mail: Jan@Wulf-Schnabel.de
an der Fachhochschule Kiel und Privatdozentin an der Universität Münster. Arbeitsschwerpunkte u.a.: Geschlecht und in der $E U$. e-mail:Uta.Klein@FH-Kiel.de Jan Wulf-Schnabel, M.A., Diplom-Sozia/wirt, Lehrbeauftragter an der Fachhochschule Kiel, Stipendiat der Hans-Böckler-Stiftung im Masterstudiengang Gender und Arbeit an der Universität Hamburg. Forschungse-mail: Jan@Wulf-Schnabel.de 
ziehen, ausbilden, lehren/beraten, informieren, gesundheitlich und sozial helfen, pflegen, medizinisch, kosmetisch behandeln. Der Typus „moderne professionelle Tätigkeitselemente" umfasst: Management, Leitungs- oder Führungstätigkeiten/Werbung, Marketing/Forschung und Entwicklung (ebd., S. 15). In seiner Auswertung der Mikrozensus-Daten 1996 zeigt sich, dass bei den personenorientierten Kerntätigkeiten Frauen leicht stärker repräsentiert sind und dass bei modernen, professionellen Tätigkeitselementen wiederum der Männeranteil höher ist (ebd., S.16).

Nach Analyse der IAB Berufsgruppe 86 (IAB 2006) findet sich der höchste Frauenanteil bei den personennahen CareTätigkeiten in der Kinderbetreuung, die in der beruflichen Ordnung die unterste Hierarchieposition einnimmt. Die Beschäftigten in den eher akademisch ausgerichteten Berufen (Sozialpädagogik und Sozialarbeit) weisen (wenngleich ebenfalls auf hohem Niveau) den relativ niedrigsten Frauenanteil auf. Hier finden sich mit aufsteigender Hierarchie vermehrt leitende und kontrollierende Tätigkeiten - und entsprechend sind verstärkt Männer zu finden.

\subsection{MÄNNER UND LEITUNGSPOSITIONEN}

Trotz des hohen Frauenanteils ist die Soziale Arbeit fest in Männerregie. Dass die wenigen Männer in den sozialen Berufen überproportional auf Steuerungs- und Leitungsebenen präsent sind, wurde schon früher in Beiträgen der Frauenforschung und vereinzelten historischen Analysen der Sozialen Arbeit thematisiert. Historisch betrachtet entwickelte sich die männliche Seite in der Sozialen Arbeit vor allem in der Führungsbürokratie und in hierarchisch leitenden Tätigkeiten (Fröschl 2001, S. 288). Bereits 1980 stellte Drake in einer der ersten Untersuchungen zum Geschlechterverhältnis in der Sozialen Arbeit fest, dass Frauen ,ihren männlichen Kollegen gegenüber benachteiligt sind: in der Entlohnung, bei Aufstiegsmöglichkeiten und durch den faktischen Ausschluss von bestimmten Arbeitsfunktionen" (Drake 1980, S. 46). Personennahe Tätigkeiten wurden von Rabe-Kleberg (1990) als vertikale, von männlicher Kontroll- und Definitionsmacht abhängige Berufs(teil)felder identifiziert, denen sich Männer meist selbst entziehen. „Die weibliche Seite ist eher auf der direkt helfenden und ,Hand anlegenden 'Seite des Berufes" (ebd., S. 65). Meinholds (1993) Betrachtung der Jugendämter in Rheinland-Pfalz erbrachte eine entsprechende Hierarchiestufung: Auf der ausführenden Ebene der SozialarbeiterInnen ermittelte sie einen Männeranteil von $31 \%$, auf der höchsten Leitungsebene waren es dann bereits $66 \%$. Die Kluft vergrößert sich mit dem Alter: Der Anteil der Männer in Leitungsfunktionen steigt, je älter sie sind, während Frauen der Aufstieg in Führungspositionen nicht gelingt. Männer in der Sozialen Arbeit profitieren offensichtlich von unsichtbaren Aufstiegsmechanismen. Frauen dagegen werden generell in Organisationen durch zahlreiche, nicht unmittelbar erkennbare Barrieren am Aufstieg in Führungspositionen gehindert - ein Phänomen, das Joan Acker (1991) als Erste mit glass ceiling (gläserne Decke) bezeichnete.

Nach den Mikrozensus-Daten von 1996 (Pich 2004) ist der Geschlechtsunterschied bei SozialarbeiterInnen und SozialpädagogInnen in Leitungsfunktionen bei UniversitätsabsolventInnen deutlich ausgeprägter als bei FachhochschulabsoventInnen. Insgesamt sind Männer unter den Angestellten mit umfassenden Führungsaufgaben oder unter höheren Beamten auffällig häufiger vertreten als Frauen. Umgekehrt muss sehr zu denken geben, dass Frauen mit einem Fachhochschulabschluss zu einem großen Teil als Erzieherinnen arbeiten. 1996 waren es $13 \%$ gegenüber 1,5 \% der Männer! In vertikaler Dimension sind es „überproportional Frauen, die keinen ihrer Ausbildung entsprechenden Beruf gefunden haben“ (ebd., S. 33).

Diese Segmentation lässt sich auch in der Lohn- und Einkommensverteilung ablesen. Nach einer Einkommensstichprobe unter SozialarbeiterInnen von Simmel-Joachim (2003) ist die Einkommensbandbreite bei Frauen deutlich größer als bei Männern. Während nur 21,5\% der Frauen über $3.000 €$ monatlich erhalten sind es 51,9\% der Männer. Der Lohnspiegel (WSI Tarifarchiv 2006) der Hans-Böckler-Stiftung verzeichnet einen geschlechtstypischen Einkommensunterschied von monatlichen $254 €$ bei SozialpädagogInnen bzw. von $231 €$ bei ErzieherInnen. ${ }^{1}$ Wir wissen inzwischen aus verschiedenen Untersuchungen, dass solche Lohnunterschiede selbst bei gleicher Humankapitalausstattung bestehen, das heißt bei gleicher Qualifikation und Berufserfahrung in derselben Jobzelle
(Hinz/Gartner 2005). Da ein Teil der Einkommensschere durch Lohnklassifizierungssysteme und damit verbundene tarifliche Eingruppierungen bedingt ist (Klein 2006a), bleibt abzuwarten, wie sich die Veränderung der Tarifstruktur im öffentlichen Dienst auswirkt.

\subsection{MEHR FRAUEN BEI GLEICH- BLEIBENDEM ARBEITSVOLUMEN}

Die genannten Lohnspiegelangaben beziehen sich auf Vollzeit-Arbeitsverhältnisse. Aufgrund der hohen Teilzeitquote bei Frauen dürfte das real zur Verfügung stehende Einkommen noch weiter auseinander liegen. Und da die bisher schon hohe Teilzeitquote der Sozialberufe beständig steigt (und dies wieder besonders bei den Berufen mit den höchsten Frauenanteilen), verschärft sich die damit verbundene Einkommensungleichheit weiter. Dies ist eine EU-weite Tendenz. Die Länder, in denen eine hohe Zunahme der weiblichen Erwerbstätigenquote zu verzeichnen ist, sind dieselben Länder, in denen die weibliche Teilzeitbeschäftigung wächst (Klein 2006a). Eine weitere Beschleunigung dieser Tendenz ist im Zusammenhang mit der europäischen Beschäftigungsstrategie zu befürchten (Klein 2006a, 2006b).

Während in Deutschland branchenübergreifend die Teilzeitquote auf $23 \%$ angewachsen ist und Frauen $85 \%$ aller Teilzeittätigen darstellen (Statistisches Bundesamt 2005b, S. 44), halten auch die (wenigen) Männer im Gesundheits-, Veterinärund Sozialwesen am Vollzeitkonzept fest (Statistisches Bundesamt 2005a). Dabei zeigt sich die Umverteilung zugunsten der Teilzeitarbeit in der Sozialen Arbeit besonders drastisch. Beispielsweise stieg die Teilzeitquote bei den SozialpädagogInnen und HeimleiterInnen in sechs Jahren (1999 zu 2005) von $26,1 \%$ auf $35,5 \%$ (plus $9,4 \%$ Punkte) und bei den KindergärtnerInnen u. ä. von $37,4 \%$ auf $49,3 \%$ (plus 11,9\%Punkte) (IAB 2006). Entgegen dem bundesdeutschen Trend wuchs hier die Anzahl der sozialversicherungspflichtig beschäftig-

\footnotetext{
Es wurden für beide Berufe die folgenden Rahmenbedingungen/Kategorien ausgewählt: ohne Leitungsfunktion, 10 Jahre Berufserfahrung, Westdeutschland, Betrieb unter 100 Beschäftigte, 38 Wochenstunden. Deutliche Lohnunterschiede gibt es aber bereits unter den Berufsanfängerinnen und Berufsanfängern (Kategorie 0-5 Jahre Berufserfahrung).
} 
ten Personen in den letzten Jahren deutlich - aber zumeist sind dies Frauen, die sich die Arbeit untereinander teilen (ebd.). ${ }^{2}$

\section{Profession und Geschlecht}

Die Positionierung von Frauen und von Männern im Berufssystem der Sozialen Arbeit kann nur im Gesamtzusammenhang der gesellschaftlichen und geschlechtshierarchischen Arbeitsteilung und der daraus resultierenden Zuweisung der "Care“Tätigkeiten an Frauen verstanden werden. $\mathrm{Zu}$ den Merkmalen der Berufe, in denen Frauen überproportional vertreten sind, zählen: begrenztere oder kürzere Laufbahnen, eine hohe Teilzeitquote, die Verberuflichung ehemals privat und unbezahlt erbrachter Arbeiten, geringere tarifliche Bezahlung trotz vergleichbarer Qualifikation. Besonders in Sozialberufen existieren Grenzschließungsprobleme, das heißt, berufliche und nicht-berufliche Arbeit sind schwer voneinander zu trennen. In diesen Bereichen werden von den Dienstleistenden enorme soziale Kompetenzen gefordert, die Gorz (1989, S. 204ff) ein „SichSelbst-Geben" nennt.

Anstelle der früher aus differenztheoretischer Perspektive vertretenen These vom vermeintlichen ,weiblichen Arbeitsvermögen", das Frauen besonders für die Arbeit in z. B. sozialen Berufen prädestiniere und ihren hohen Anteil erkläre, rückt die Geschlechterforschung heute die „Vergeschlechtlichung von Berufen" in den Vordergrund. Daher sollte auch ein Begriff wie „Frauenberuf" mit Vorsicht verwendet werden, denn auch er treibt die Vergeschlechtlichung voran. Der Verweis auf eine vermeintlich besondere Eignung von Frauen für den sozialen Bereich wird gerne als Legitimation der ungleichen Geschlechterordnung genutzt und ging immer mit einer Entwertung der weiblichen Berufsarbeit einher. Vor diesem Hintergrund sind Wandlungsprozesse im Feld der Sozialen Arbeit in ihrer Wirkung auf Geschlechterverhältnisse interessant. Der Rückgriff auf eine vermeintliche Geschlechterdifferenz verliert gesellschaftlich an Legitimität, es ist allgemein zu einer „Ablösung einer Semantik der Differenz durch ein Modell der Gleichberechtigung" gekommen (Heintz 2001). Dies könnte zusammen mit den Professionalisierungsbestrebungen (Ver- wissenschaftlichung, Benennung erforderlicher Kompetenzen, fachliche Differenzierung und Spezialisierung, Methodenboom, Qualitätsmanagement) in den Berufen der Sozialen Arbeit zu einer gewissen "Entgeschlechtlichung" auch in den Leitungspositionen führen. Es spricht einiges für die These, dass die Akteurinnen und Akteure in ihrem Selbstverständnis zunehmend weniger die Geschlechtsbezogenheit der Fähigkeiten hervorheben, sondern deren professionelle Seite. Entsprechende Andeutungen ergeben sich aus den vorläufigen Ergebnisse einer Organisationsanalyse (Wulf-Schnabel 2005): Demnach ist der Frauenanteil auf der Managementebene eines Verbandes im Verlauf eines umfassenden Reorganisationsprozesses klar gestiegen.

Insgesamt ist für die Sozialberufe eine Niveauerhöhung festzustellen: durch einen Anstieg der Fachkräfte bei gleichzeitigem Rückgang Ungelernter, durch eine Zunahme von Fachhochschulstudiengängen und durch eine Differenzierung universitärer Studiengänge (Karsten 2000, S. 85). Professionalisierungsbemühungen gehen üblicherweise mit der Aufwertung von Berufen einher, weshalb hier auch die Chancen für einen steigenden Männeranteil in den sozialen Berufen insgesamt und besonders in den personennahen Ebenen gesehen werden. Hier besteht die Gefahr, dass die Professionalisierungsbestrebungen vergeschlechtlicht verlaufen, indem die neue Professionalität dem „weiblich unprofessionellen" dichotom gegenüber gestellt wird. So ist zu untersuchen, ob Tätigkeitselemente, die oben als moderne, professionelle Elemente gefasst wurden, im Diskurs vermännlicht werden. Eine männliche Konnotation von Konzepten wie Ökonomisierung, betriebswirtschaftliche Orientierung, Managementkompetenzen ist nicht von der Hand zu weisen. In der Ausübung der Berufe stellt sich die Frage nach den „Doing gender-Prozessen“. Untersuchungen, beispielsweise über Pflegeberufe (Ummel 2001; Heintz 2001), machen deutlich, dass Männer die Differenz zwischen Männern und Frauen betonen und ihre „besondere" Eignung als Mann überhöhen. Auch ist offensichtlich, dass Frauen selber Männer auf geschlechtstypische Tätigkeiten festlegen und verweisen. ${ }^{3}$ Im Kindertagesstättenbereich sind dies die berühmten Hausmeistertätigkeiten; in der Pflege vor allem Tätigkeiten mit hohem Krafteinsatz. Doch wie entwickelt sich der Männeranteil tatsächlich? Um Aussagen zum künftigen Berufseintritt zu erhalten und daraus eine Prognose für die Arbeitswelt ableiten zu können, werden im Folgenden die Hochschulstudiengänge analysiert.

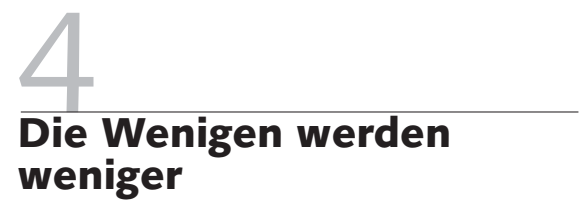

Wie sieht das Geschlechterverhältnis bei den Studierenden in den Studiengängen Sozialpädagogik, Sozialarbeit und Sozialwesen aus? Gegenwärtig liegt es bei $76 \%$ (Frauen) zu $24 \%$ (Männer) (Statistisches Bundesamt 2005c). Bei der Zeitreihenanalyse (Abbildung 1$)^{4}$ fällt der steigende Männeranteil Mitte bis Ende der 1970er Jahre auf. Er nahm im Studiengang Sozialpädagogik Mitte der 1970er Jahre sogar sprunghaft die $40 \%$-Marke und lag damit um bis zu 19 \%-Punkte höher als heute. Dieser vorübergehend gestiegene Männeranteil korrespondiert einerseits mit der politisch geprägten Phase der 1970er Jahre, durch die verstärkt Männer in die Soziale Arbeit gelangten - die Politisierung durch die Studentenbewegung erreichte insbesondere den Studiengang der Sozialpädagogik (Fröschl 2001, S.291f.). Andererseits vollzog sich genau in dieser Periode die Überführung der höheren Fachschulen in Fachhochschulen, das heißt, es erfolgte eine hohe Aufwertung des Berufsbildes. Allerdings war diese Entwicklung nur kurz-

2 Nach eigenen Berechnungen auf Basis der IAB Daten (IAB 2006) stieg in der Berufsordnung 864 (Kinderbetreuung, Erziehung) der Beschäftigungsindex von 1999 bis 2005 um 11 Punkte, der Frauenindex um 10 Punkte und der Teilzeitindex um 46 Punkte. Der Beschäftigungsindex in der Berufsordnung 861 (Sozialarbeit) stieg im Zeitraum um 19 Punkte, der Frauenindex um 20 Punkte und der Teilzeitindex um 49 Punkte. Ebenso eng wachsen Beschäftigungsindex (plus 18 Punkte) und Frauenindex (plus 20 Punkte) in der Berufsordnung 862 (Sozialpädagogik), und mit 61 Punkten Zuwachs liegt der Teilzeitindex deutlich darüber. Die Steigerung der Teilzeitquote liegt über dem Wachstum der sozialversicherungspflichtigen Beschäftigungsverhältnisse und über dem Wachstum der Frauenerwerbsquote.

3 Arbeiten zu "boundary work" untersuchen "doing gender while doing work " von Frauen und Männern in geschlechtsuntypischen Berufen.

4 Die Abbildungen 1-3 basieren auf eigenen Berechnungen mittels Daten des Statistischen Bundesamtes (2005b), wobei jeweils die Datenreihen zum Wintersemester (WS) ausgewertet wurden. 
fristiger Art, denn bereits Ende der 1970er Jahre fiel der Männeranteil unter das Ausgangsniveau zurück.

Knapp 14 Jahre später stieg der Männeranteil wieder, und dieses Mal im Studiengang Sozialpädagogik und im Studiengang Sozialarbeit. Doch auch dieser Anstieg hielt sich nicht, und insgesamt ist eine sich deutlich verschärfende Geschlechtertrennung auffällig, wie die lineare Trendberechnung zeigt. ${ }^{5}$

Der insgesamt abnehmende Männeranteil hat nicht nur eine relative Bedeutung, wie Abbildung 2 zeigt. Offenkundig waren Frauen zunehmend interessiert, Soziale Arbeit zu studieren. Und so konnten sie den wachsenden Arbeitskräftebedarf abdecken. Dies war bei Männern nicht annähernd der Fall.

Der Anteil der Männer bewegt sich wellenförmig. Er steigt und fällt, jedoch nur etappenweise und sehr begrenzt. Es wird verschiedentlich betont, dass heute insgesamt mehr Männer als vor 30 Jahren im Sozialen Sektor tätig sind. Bedenkt man jedoch die deutsch-deutsche Wiedervereinigung und die Entwicklung ab 1990, ergibt sich ein ganz anderes Bild. Die über dem Trend liegende Entwicklung zwischen 1993 und 1999 passt auffällig gut zu der Ausweitung der Studienplätze in den neuen Bundesländern. In der Zeit von 1993 bis 2000 erhöhte sich die Anzahl der Studierenden in den neuen Bundesländern für den Bereich Soziale Arbeit von rund 3.000 auf ca. 8.000 (Statistisches Bundesamt 2005c). Gegenüber dieser Steigerung studierten bundesweit nur 417 mehr Männer, aber immerhin 7.316 mehr Frauen an den Hochschulen (Anstieg Wintersemester 1993/94 zu 2000/01). Statistisch ist also die Bevölkerungs-, Gebiets- und Studienplatzzunahme im Spiegel der deutsch-deutschen Vereinigung bedeutsamer, sodass in Gesamtdeutschland anteilig weniger Männer Soziale Arbeit studierten. ${ }^{6}$ Offenkundig fanden stets nur wenige Männer den Weg in die Soziale Arbeit. Weil die Soziale Arbeit seit den 1970er Jahren einen regelrechten Boom erlebte, sind mittlerweile immer mehr Personen von der Segregationslage betroffen und werden von ihr unmittelbar geprägt - und zwar Beschäftigte (Multiplikatoren) wie auch die AdressatInnen Sozialer Arbeit.

Wird die Entwicklung der letzten Jahre aus der Perspektive der Studienfächer betrachtet, ergeben sich noch ganz andere Aspekte (Abbildung 3). Um den auffälligen

Abb. 1: Männeranteile in den Studiengängen Sozialer Arbeit - in \% -

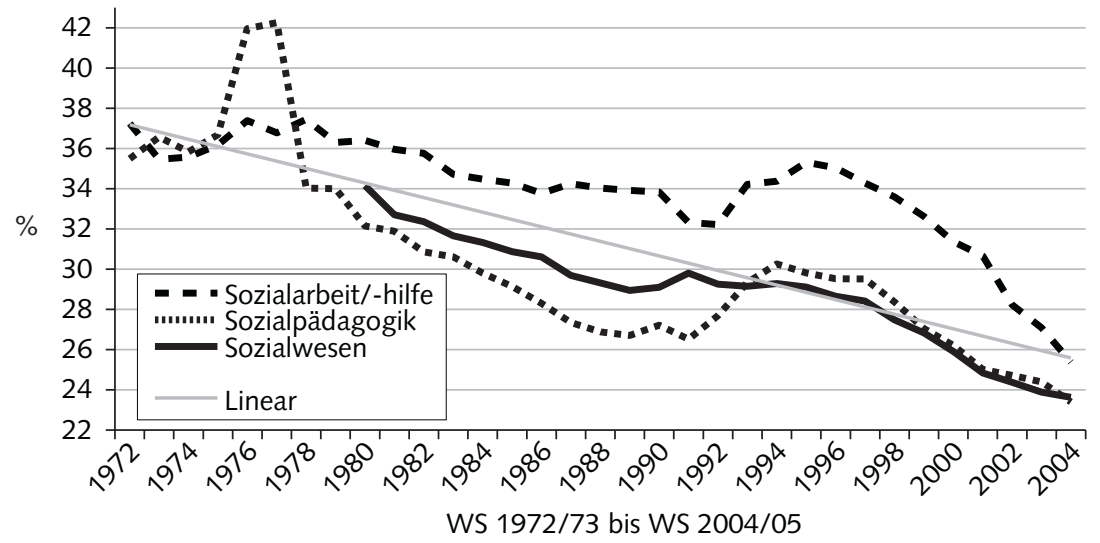

Quelle: Berechnungen der Autoren auf Basis Statistisches Bundesamt 2005c

WSI Hans Böckler

\section{Abb. 2: Segregationsschere - Entwicklung der Anzahl von Frauen/ Männern in den Studiengängen Sozialer Arbeit}

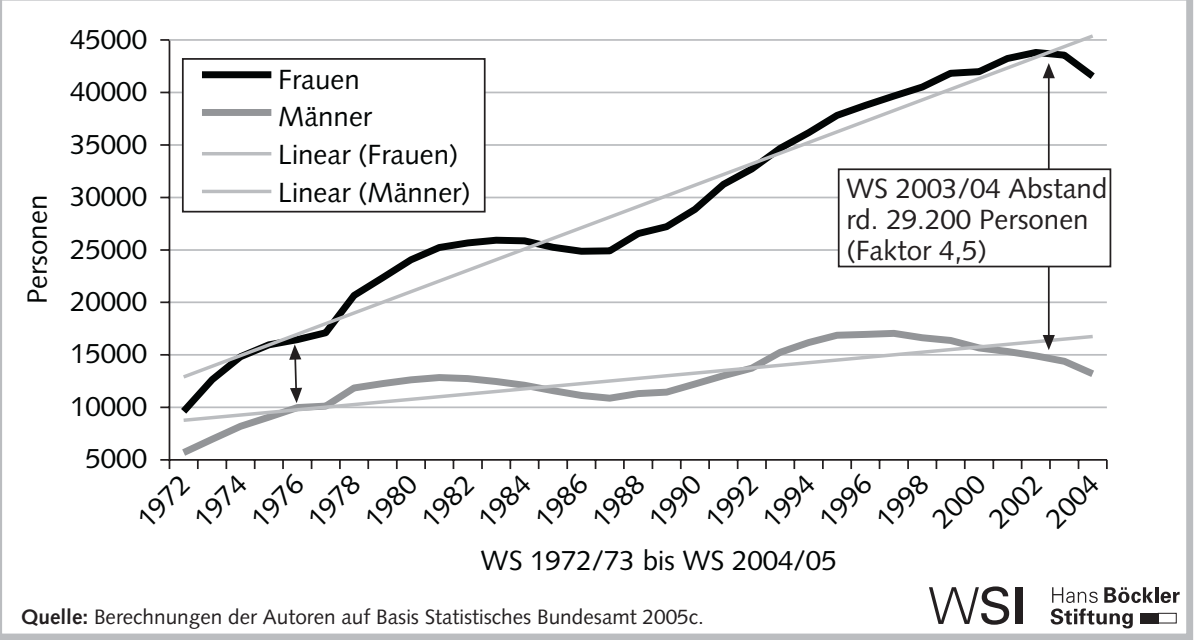

Männeranstieg in den 1990er Jahren genauer zu untersuchen, wurde der größte Anstiegswinkel berechnet und dort die Ausgangslage von Frauen und Männern auf die Index-Basis 100 gesetzt. Wird nun das Wachstum der Anzahl der Studierenden verglichen, so zeigt sich ein recht erstaunliches Bild: Männer legten in den Studiengängen der Sozialen Arbeit sogar stärker zu als Frauen.

Aber nach dem Wintersemester 1997/ 98 reißt dieser Trend ab und kehrt sich um. Zur genaueren Untersuchung wurden die jeweiligen Studiengänge für sich betrachtet. Dabei wird deutlich, dass Männer mit ihrer Studienfachwahl auf das falsche Pferd gesetzt haben bzw. wurden. Insbesondere im Studiengang Sozialpädagogik haben Männer zunächst ihre Anzahl gesteigert, aber gerade dieser Studiengang hat an Be- deutung verloren. Heute studieren so wenige Männer (in absoluten Zahlen) Sozialpädagogik wie zuletzt vor 27 Jahren, das heißt vor dem Boom der Sozialen Arbeit.

\footnotetext{
5 Der reformierte Studiengang Sozialwesen entstand Anfang der 1980er Jahre. Der Abschluss kann Diplom-Sozialpädagogik bzw. Diplom-Sozialarbeit bedeuten, dies ist je nach Hochschule unterschiedlich. Auf Nachfrage teilte das Statistische Bundesamt mit, dass in den Daten neuere Studiengänge (z. B. Sozialmanagement) enthalten sind, aber die Bachelor-/ Master-Umstellung noch nicht berücksichtigt wurde.

6 2000/01 verteilten sich die Studierenden (Soziale Arbeit) auf rund 49.400 West, 7.800 Ost, 3.900 Berlin; 1993/94 waren es ca. 45.700 West, 2.700 Ost, 2.600 Berlin. Selbst unter der Annahme, dass in den neuen Bundesländern nur etwa $10 \%$ der Studierenden Männer sind, würde sich ihre absolute Zahl mit rund 500 Männern stärker erhöhen als die bundesweite Zahl im Vergleichszeitraum.
} 


\section{Abb. 3: Index Entwicklung: Anzahl Frauen/Männer in den Studiengängen Sozialer Arbeit (WS 1991/92 = 100)}

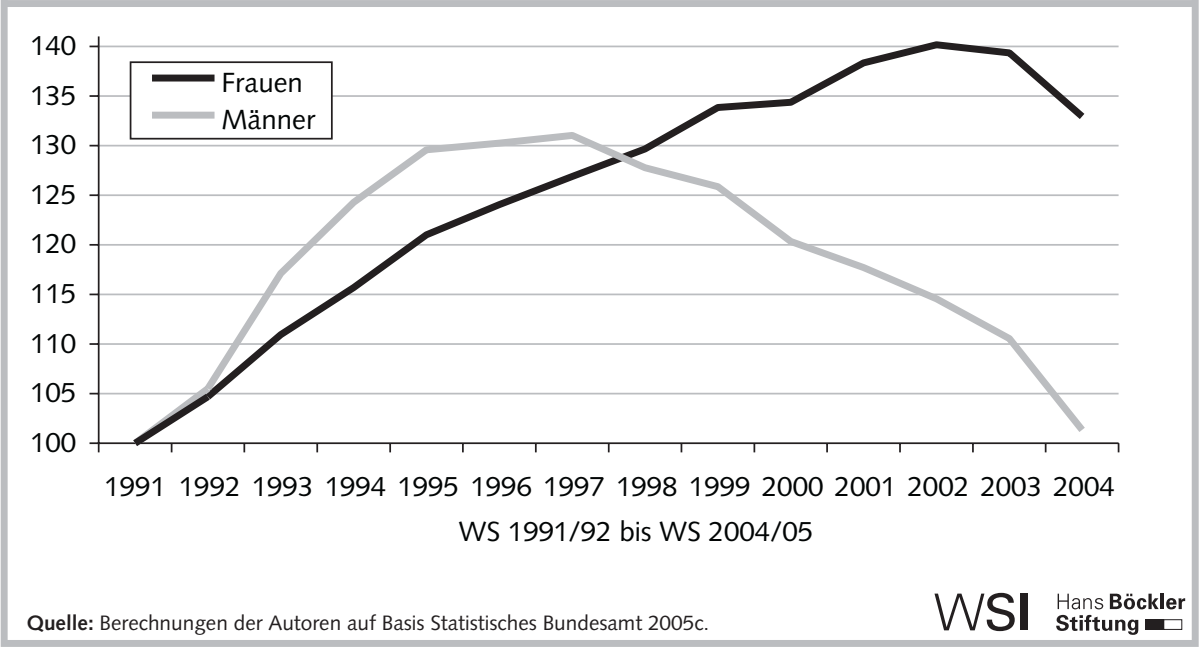

Dagegen hat der Studiengang Sozialwesen absolut die größte Bedeutung - und genau hier findet sich der höchste Abstand zwischen Männern und Frauen.

$\mathrm{Zu}$ den rückläufigen Männeranteilen kommt der geringere Bildungserfolg der Männer hinzu. Generell haben seit den 1980er Jahren die Frauen die Männer im Bildungserfolg ein- und mittlerweile überholt - und dies EU-weit (Klein 2006a). Im Studium der Sozialen Arbeit ist der Bildungsvorsprung deutlich: Während 2002 immerhin 81,5 \% der Frauen ihr Studium in FH-Studiengängen Sozialer Arbeit erfolgreich beendeten (bezogen auf das Anfängerjahr 1997), gelang dies nur $65 \%$ der Männer (ISA 2005).

\section{Fazit}

Studieren also immer weniger Männer Soziale Arbeit, weil sie mit den Frauen nicht mithalten können und so ihr Führungsanspruch neuerdings verloren geht? Tatsächlich ist die Zahl der weiblichen Studienanfänger in den vergangenen Jahren in Deutschland stärker gestiegen als die der männlichen, ${ }^{7}$ sodass 2002 erstmals etwas mehr Frauen als Männer ein Studium begonnen haben. Damit sind die Geschlechterverhältnisse insgesamt zwar noch nicht ins Wanken geraten, allerdings bekommen Männer zunehmend Karrierekonkurrenz von qualifizierten Frauen.

Die von uns beschriebene, seit Etablierung der Fachhochschulen nie da gewesene Segregationsdynamik verschiebt sich gegenwärtig und künftig auf den Arbeitsmarkt und verändert das dortige Geschlechterverhältnis. Der abnehmende Männeranteil in den Studiengängen der Sozialen Arbeit könnte also auch Auswirkungen auf die Führungsebenen haben. Entgegen der naheliegenden Vermutung steigern Männer unter den Bedingungen eines zunehmenden Wettbewerbs- und Arbeitsmarktdrucks in der Sozialen Arbeit ihren Anteil nicht zulasten der Frauen. Im Gegenteil: Frauen breiten sich im boomenden Sozialdienstleistungssektor aus. Insgesamt wächst sowohl die Anzahl der abhängig Beschäftigten als auch der Abstand im Frauen-Männer-Verhältnis bei den Studierenden - und dies seit Jahrzehnten. So kann das 20. Jahrhundert - in Anlehnung an Rauschenbach (1999) - als ein sozialpädagogisches Frauenjahrhundert bezeichnet werden. Allerdings reduziert sich die Arbeitszeit insbesondere bei Frauen immer mehr, sodass ihr tatsächliches Arbeitszeitvolumen nur schwer abschätzbar ist. Männer können oder wollen in der Sozialen Arbeit nicht Fuß fassen, denn was Frauen gelingt, fällt Männern schwer: qualifiziert, teilzeit und personennah zu arbeiten.

Bedingt durch den Rückzug des Sozialstaates befinden sich die Ausbildungs- und Arbeitsbedingungen der Sozialen Arbeit unter massivem Veränderungsdruck. Einerseits ist eine enorm gestiegene fachliche Spezialisierung erkennbar, und ehemals rein berufliche Felder werden für akademische Qualifikationen durchlässiger. Außerhalb des Hochschulwesens erworbene Kenntnisse und Fähigkeiten können formal mittlerweile bis zu $50 \%$ eines Hochschulstudiums ersetzen (KMK 2002). So werden künftig z. B. ErzieherInnen ihre beruflichen Qualifikationen in Bachelor-Studiengänge einbringen. Die Differenzierung und Zunahme neuer Studiengänge (Bachelor-/Master-Programme) erfordern eine Intensivierung der Forschung, die sich nicht allein aus anderen Human- oder Sozialwissenschaften speist, sondern die aus der eigenen Profession herauskommen muss. Mit der Akademisierung sozialer Berufe wird eine höhere Qualifizierung erreicht, und mit der Modularisierung der Hochschulstränge entsteht ein dezentrales Repertoire unterschiedlicher Bildungsbausteine. Damit erhöht sich auch die Chance, dass der Abschluss nicht für einen eng umrissenen Beruf qualifiziert, vielmehr Anknüpfungspunkte für verschiedene Berufsfelder bietet und zudem für individuelle Profilbildungen nutzbar ist.

Andererseits sind nicht nur die Qualitätsanforderungen gestiegen, sondern es steigt auch der quantitative Bedarf nach sozialen Dienstleistungen. Allerdings wird der wachsende Bedarf nicht adäquat öffentlich finanziert, sodass Teile Sozialer Arbeit (wieder) privat erbracht werden (müssen) oder ehrenamtlich substituiert werden (müssen). Dazwischen schweben neue und alte Formen hilfsberuflicher Arbeit. In der Kinderbetreuung wird dies deutlich, wenn ErzieherInnen durch den relativ neuen und tariflich deutlich günstigeren Hilfsberuf „Sozialpädagogische Assistenz" ersetzt werden.

Wird diese Entwicklung mit der steigenden Segregation Sozialer Arbeit in Verbindung gebracht, ergeben sich Konsequenzen für das Selbstverständnis und für das Profil Sozialer Arbeit. In Teilsegmenten geht es von der umfassenden fachlichen Zuständigkeit hin zu eher ausführenden Tätigkeiten, die des Gesamtverständnisses Sozialer Arbeit beraubt sind. Anhand der ausgewiesenen Datenlage ist absehbar, dass der steigende soziale Dienstleistungsbedarf von Frauen erbracht wird. Durch diesen wachsenden Multiplikatoreneffekt wird die unmittelbare Soziale Arbeit noch stärker weiblich konnotiert. Gleichzeitig gelingt es anderen Frauen, sich auf den höheren Ebenen Sozialer Arbeit zu etablieren. Hier entsteht ein Managerialismus, um niedere,

\footnotetext{
7 Gemessen an ihren schulischen Bildungserfolgen ist ihr Anteil unter den StudienanfängerInnen aber weiterhin unterproportional (Bothfeld et al. 2005, S. 89).
} 
ausführende Teilsegmente zu koordinieren und zu überwachen (Lindenberg 2004, S. 8f.). Nur: Wo bleiben da die Männer? Dass die Männer sich von der Sozialen Arbeit immer stärker verabschieden, konnte mit diesem Beitrag hoffentlich verdeutlicht werden. Doch es gibt weiteren Klärungsbe- darf: Ist der Weg der Männer aus der Sozialen Arbeit als eine vorausschauende Flucht vor Statusverlust zu deuten, weil die geringe gesellschaftliche Anerkennung Sozialer Arbeit noch geringer wird? Oder gibt es andere Faktoren, die explizit Männer an Sozialer Arbeit hindern? Um dies heraus zu finden, versuchen wir in einem Forschungsprojekt erstens die Professionalisierungsstrategien Sozialer Arbeit zu analysieren und zweitens die Anforderungen an die Arbeitskräfte in ihren Wirkungen auf die Geschlechterverhältnisse zu bestimmen.

\section{LITERATUR}

Acker, J. (1991): Hierarchies, Jobs, Bodies: A Theory of Gendered Organizations, in: Lorber, J./Farrell, S. A. (Eds.), The Social Contruction of Gender, Newbury Park

Bothfeld, S./Klammer, U./Klenner, C./Leiber, S./Thiel, A./Ziegler, A. (2005): WSI-FrauenDatenReport 2005 - Handbuch zur ökonomischen und sozialen Situation von Frauen, Berlin

Bundesministerium für Familie, Senioren, Frauen u. Jugend (BMFSFJ)

(Hrsg.) (2005): Datenreport zur Gleichstellung von Frauen und Männern in der Bundesrepublik Deutschland, Berlin

Cloos, P./Züchner, I. (2005): Das Personal der Sozialen Arbeit. Größe und Zusammensetzung eines schwer zu vermessenden Feldes, in: Thole, W. (Hrsg.): Grundriss Soziale Arbeit. Ein einführendes Handbuch, Opladen, S. 711-730

Drake, H. (1980): Frauen in der Sozialarbeit. Sexismus - die geschlechtsspezifische Diskriminierung, Neuwied/Darmstadt

Fröschl, E. (2001): Beruf Sozialarbeit, in: Gruber, C./Fröschl, E. (Hrsg.): Gender-Aspekte in der Sozialen Arbeit, Wien, S. 285-308

Gorz, A. (1989): Kritik der ökonomischen Vernunft, Berlin Heintz, B. (2001), Geschlecht als (Un-)Ordnungsprinzip. Entwicklungen und Perspektiven der Geschlechtersoziologie, in: Dies. (Hrsg.). Geschlechtersoziologie, Kölner Zeitschrift für Soziologie und Sozialpsychologie, Sonderheft 41, S. 9-29

Hinz, T./Gartner, H. (2005): Lohnunterschiede zwischen Frauen und Männern in Branchen, Berufen und Betrieben, IAB DiscussionPaper 4 Institut für Arbeitsmarkt- und Berufsforschung (IAB) (2006): Berufe im Spiegel der Statistik. Beschäftigung und Arbeitslosigkeit 1999-2005. http://www.pallas.iab.de/bisds/berufe.htm, Stand der Recherche: 20.09.06

Informationssystem Studienwahl \& Arbeitsmarkt (ISA) (2005): Sozialwesen: Sozialarbeit und Sozialpädagogik. http://www.uni-essen.de/isa/ fg_sozial_gesund/sozialwesen/sozialwesen_hs_frm.htm, Stand der Recherche: 26.09 .05

Karsten, M.-E. (2000): Personenbezogene Dienstleistungen für Frauen. Aktuelle Tendenzen und Professionalisierungserfordernisse, in: Friese, $M$. (Hrsg.): Modernisierung personenorientierter Dienstleistungen, Opladen, S. $74-87$
Kasiske, J./Krabel, J./Schädler, S./Stuve, O. (2006): Zur Situation von Männern in „Frauen-Berufen“ der Pflege und Erziehung in Deutschland. Eine Überblicksstudie, in: Krabel, J./Stuve, O. (Hrsg.): Männer in „Frauen-Berufen“ der Pflege und Erziehung, Opladen, S. 11-110

King, V. (2000): Entwürfe von Männlichkeit in der Adoleszenz, in: Bosse, H./King, V. (Hrsg.): Männlichkeitsentwürfe. Wandlungen und Widerstände im Geschlechterverhältnis, Frankfurt/Main New York, S. 93-107 Klein, U. (2006a): Geschlechterverhältnisse und Gleichstellungspolitik in der Europäischen Union, Lehrbuch, Wiesbaden

Klein, U. (2006b): Europäische Integration und Geschlechterverhältnisse, in: Böllert, K./Hansbauer, P./Hasenjürgen, B./Langenohl, S. (Hrsg.): Zur Produktivität des Sozialen - Den sozialen Staat aktivieren, Wiesbaden Kultusministerkonferenz (KMK) (2002): Anrechnung von außerhalb des Hochschulwesens erworbenen Kenntnissen und Fähigkeiten auf ein Hochschulstudium. Beschluss der Kultusministerkonferenz vom 28.06.2002. http://www.kmk.org/doc/beschl/anrechnung.pdf, Stand der Recherche: 22.11.2006

Lindenberg, M. (2004): Manage mich! Sieben Thesen zur Zukunft Sozialer Arbeit im aktivierenden Staat, in: FORUM für Kinder- und Jugendarbeit 3, Verband Kinder- und Jugendarbeit e. V., Hamburg, S. 4-13 Meinhold, M. (1993): Sozialarbeiterinnen, Frauenkarrieren, Münster Pich, W. (2004): Sozialarbeiter/innen und Sozialpädagogen/innen im Mikrozensus 1996. Manuskript eines Vortrags auf der 3. Mikrozensusnutzerkonferenz am 9.10. 2003 in Mannheim

Rabe-Kleberg, U. (1990): Sozialer Beruf und Geschlechterverhältnisse. Oder: Soziale Arbeit zu einem Beruf für Frauen machen!, in: Cremer, C./Bader, C./Dudeck, A. (Hrsg.): Frauen in sozialer Arbeit. Zur Theorie und Praxis feministischer Bildungs- und Sozialarbeit, Weinheim/München, S. 60-71

Rabe-Kleberg, U. (2004): Feminisierung der Erziehung von Kindern. Chancen oder Gefahr für die Bildungsprozesse von Mädchen und Jungen? Erkenntnisse, Argumente, Materialien. Expertise im Rahmen des 12. Kinder- und Jugendberichts

Rauschenbach, T. (1999): Das sozialpädagogische Jahrhundert. Analysen zur Entwicklung sozialer Arbeit in der Moderne, Weinheim 
Rohrmann, T. (2006): Männer in Kindertageseinrichtungen und Grundschulen: Bestandsaufnahme und Perspektiven, in: Krabel, J./Stuve, O. (Hrsg.): Männer in „Frauen-Berufen“ der Pflege und Erziehung, Opladen, S. 111-133

Simmel-Joachim, M. (2003): Karriere in der Sozialen Arbeit? - nein danke! Kurzfassung der Ergebnisse einer Umfrage zu Berufseinstieg und -verlauf ehemaliger Studierender des Fachbereichs Sozialwesen der FH Wiesbaden im Sommersemester 2002, Wiesbaden

Statistisches Bundesamt (2005a): Sozialversicherungspflichtig Beschäftigte im Gesundheits-, Veterinär- und Sozialwesen, GENISIS-Online, 30.06. Statistisches Bundesamt (2005b): Leben und Arbeiten in Deutschland. Ergebnisse des Mikrozensus 2004, Wiesbaden
Statistisches Bundesamt (2005c): Studierende in Deutschland. Zusammenstellung der Fachserie 11/Reihe 4.1, auf Anfrage

Ummel, H. (2001): Andere Männer im „anderen“ Beruf? Umbrüche und Persistenzen im Geschlechts-Selbstverständnis von Pflegern, in: Döge, P./Meuser, M. (Hrsg.): Männlichkeit und soziale Ordnung. Neue Beiträge zur Geschlechterforschung, Opladen, S. 159-181

WSI-Tarifarchiv der Hans-Böckler-Stiftung (2006): Lohnspiegel, Lohnund Gehaltscheck. Internet: http://www.lohnspiegel.org/index.php? pid =46, Stand der Recherche: 02.08.2006

Wulf-Schnabel, J. (2005): Die Reorganisation Sozialer Arbeit in ihrer Bedeutung für das Geschlechterverhältnis auf der Managementebene Am Beispiel eines Landeswohlfahrtverbandes, Universität Hamburg, unveröffentlicht

\section{Öffentliche Dienstleistungen unter Privatisierungsdruck}

Die Reorganisation der öffentlichen Infrastruktursektoren in den Mitgliedsstaaten der Europäischen Union

Gemeinsame Konferenz des

Wirtschafts- und Sozialwissenschaftlichen Institutes (WSI) in der Hans-Böckler-Stiftung und der Forschungsgruppe Europäische Integration (FEI) an der Philipps-Universität Marburg

\section{9./30. Juni 2007 in Marburg/Lahn}

Was sind die Auswirkungen von Privatisierung und Liberalisierung öffentlicher Dienstleistungen in Europa? Auf der Konferenz werden neben Vorträgen zu politökonomischen Triebkräften und Instrumenten der Reorganisation öffentlicher Dienstleistungen auf EU-Ebene vier parallele Workshops zur Liberalisierung und Privatisierung im Post-, ÖPNV-, Krankenhaus- und Energiesektor im europäischen Vergleich durchgeführt. Auswirkungen auf Arbeitsbedingungen und Arbeitsbeziehungen werden beleuchtet und politische Strategien und Initiativen mit WissenschaftlerInnen, GewerkschaftlerInnen und VertreterInnen aus sozialen Bewegungen aus nationaler und internationaler Perspektive diskutiert.

Vorträge, Workshops und Diskussionen u.a.

- Europäische Initiativen und Instrumente zur Reorganisation öffentlicher Dienstleistungen (Klaus Dräger, EP)

- Trends im europäischen Privatisierungsprozess - Projektergebnisse

(Hans-Jürgen Bieling/Christina Deckwirth/Stefan Schmalz, FEI)

- Auswirkungen der Privatisierung auf Arbeitsbedingungen und Arbeitsbeziehungen

(Thorsten Schulten/Torsten Brandt,WSI)

- Gewerkschaftliche Handlungsoptionen und Proteste sozialer Bewegungen

(Werner Sauerborn, ver.di Stuttgart; Alexandra Strickner, Attac Österreich; Volker Mittendorf, Forschungsstelle Bürgerbeteiligung und Direkte Demokratie Marburg)

- Perspektiven der „mixed economy“ und des öffentlichen Sektors in Europa - Abschlussdiskussion

(Frank Deppe, FEI; Alexandra Strickner, Attac Österreich; Klaus Dräger, EP; Richard Pond, EGÖD)

Anmeldung/Information: Kathrin Dreews, FEI, e-mail: Drewsk@students.uni-marburg.de 